\title{
Plasmonic Bandgaps in $1 D$ Arrays of Slits on Metal Layers Excited by Out-of-Plane Sources
}

\author{
Roberto Marani, Marco Grande, Vincenzo Petruzzelli, and Antonella D'Orazio \\ Dipartimento di Elettrotecnica ed Elettronica, Politecnico di Bari, Via E. Orabona 4, 70125 Bari, Italy \\ Correspondence should be addressed to Antonella D’Orazio, dorazio@poliba.it
}

Received 28 March 2012; Accepted 16 May 2012

Academic Editor: Zhaolin Lu

Copyright ( $) 2012$ Roberto Marani et al. This is an open access article distributed under the Creative Commons Attribution License, which permits unrestricted use, distribution, and reproduction in any medium, provided the original work is properly cited.

\begin{abstract}
We analyze the effective opening of finite bands of inhibited transmission in realistic systems excited by actual out-of-plane sources. We first observe how the excitation of surface plasmon polaritons in one-dimensional arrays of metal slits depends on the angle of incidence of the source field. Then, the well-known grating-coupling equation is revised in order to find an asymmetric structure with equivalent parameters which, under perfectly normal excitation, is able to exhibit surface plasmon polariton modes at the same wavelengths of the original structure which undergoes a nonorthogonal incidence of the light. In this way we demonstrate through finite-element simulations that a realistic system, probed by a source beam in a finite light-cone, can be effectively decomposed in several equivalent systems with different physical and geometrical parameters, with results in the enlargement of the theoretically expected punctual minimum of transmission.
\end{abstract}

\section{Introduction}

In the last years, the excitation of collective oscillations of free electrons over metal interfaces has been deeply investigated in literature since it allows the successful control of light in terms of localization and enhancement of electromagnetic fields. As already known, the free-electron oscillations can assume several forms, ranging from localized surface dielectric oscillations on metal nanoparticles (localized surface plasmons, LSPs) to freely propagating electron density waves on metal surfaces (surface plasmon polaritons, SPPs). Surface plasmons show unique properties when they propagate over metal gratings and interact with free waves in the dielectric surroundings. The results of the fundamental research in the area of plasmonics have demonstrated that SPPs and LSPs can be exploited for many potential applications residing in sensing [1-5], light manipulation $[6,7]$ and emission [8-10], energy conversion [11-13], and optical nanoantennas [14, 15], just to mention a few.

Many researchers have proved that under particular conditions many effects of enhanced and inhibited transmission emerge. For instance, subwavelength apertures carved on metal layers provide extraordinary optical transmission
(EOT) caused by the resonant excitation of SPP modes over the input and the output interfaces between the metal and the dielectric $[16,17]$. The in-phase cooperation among modes allows light beams impinging far from the apertures to assist the spectral response with results in normalizedto-area transmissions higher than one. Moreover, a second mechanism of resonance starts arising when the input wavelength is able to excite waveguide modes within the slits which can be exploited to carry energy within the aperture along a direction perpendicular to the grating wavevector, but in the grating plane (gap plasmon, GP) [18]. Onedimensional gratings allow also the existence of minima of transmission, whose properties are directly linked to the geometrical and physical parameters of the whole systems [19]. Moreover, this resonant behavior, due to the formation of propagating SPP modes over the metal-dielectric interface, can be found even in two-dimensional arrays of metal objects [20]. Without loss of generality, the incident electromagnetic field can be decoupled in two components along the grating directions, each one experiencing its own periodicity, so that a two-dimensional array can be simplified in two different one-dimensional gratings, depending on the polarization of the source. 
All the considerations made for arrays of slits face with practical problems due to the splitting of surface modes when the system is illuminated by a source with nonorthogonal incidence. As demonstrated with the experimental analysis in [4], whenever a realistic measurement is performed over arrays of metal objects illuminated by white light lamp, the resulting resonance giving inhibited transmission is constituted by a weighted superposition of resonances in a cone of allowed angles of incidence depending on the numerical aperture of the microscope objective used in the optical setup. In other words the expected single resonance broadens since many SPP modes are excited over the metaldielectric interface, giving rise to an enlargement of the plasmonic bandgap. This feature is useful for applications in thin-film solar cell technology, where such structures are required to act as broadband reflectors for source field impinging with arbitrary incidences.

In this paper we give evidence to the formation of an effective plasmonic bandgap in an array of sub-wavelength slits on a metal layer excited by an out-of-plane actual source. In order to achieve this goal, we start from the analysis of the theoretical effects of the presence of a tilted source on the excitation of SPP modes. Then we define an equivalence of structures able to convert such modes in spatially separated SPP resonances, with the same spectral properties of the original ones, but excited through the application of an orthogonal source. Since the equivalent parameters depend on the angle of incidence of the source field of the original structure, we observe that a practical system, excited by an input beam in a finite light-cone, has a spectral behaviour comparable to the one obtained by a superposition of results coming out by the investigation of several equivalent structures probed by perfectly orthogonal sources. Thus, we demonstrate the effective opening of a finite bandgap in actual systems, although a single minimum of transmission is expected.

The paper is organized as follows: in Section 2 we will show the basis of the analysis of one-dimensional arrays of sub-wavelength slits on metal layers in both symmetric and asymmetric configurations and under different angles of incidence; then, Section 3 will be dedicated to the definition of the parameters of the equivalent structure, together with a comparison of results with those of the original arrangement; conclusions and remarks will be finally reported in Section 4 .

\section{One-Dimensional Arrays of Slits}

A sketch of the structure considered in this paper is reported in Figure 1. It consists of an infinite one-dimensional array of slits carved on a gold thin film. As shown in Figure 1, the geometrical parameters defining the structure are the metal thickness $w$, the grating period $p$, and the width of the strips $l$, whereas $a$ is the extension of the sub-wavelength gaps, easily equal to the difference between the grating period and the strips width. In this first part of analysis, the structure is assumed to be placed in a homogeneous environment made of air.

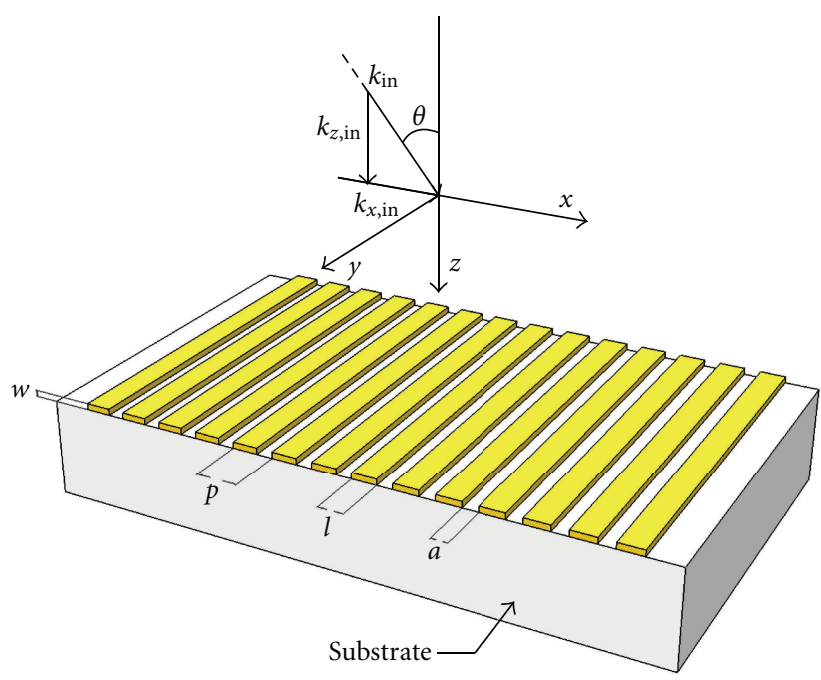

FIgURE 1: Sketch of an infinite one-dimensional array of metal strips, $(l \times w)$ sized, $p$ is the period of the array, and $a$ is the subwavelength extension of the slits.

Maxwell's equations for the propagation of electromagnetic waves have been solved via a finite-element commercial code [21] applied on a computing domain bounded by periodic boundary conditions along the $x$-direction in Figure 1 and perfectly matched layers in the $z$-direction. Since Maxwell's equations are rigorously solved depending on the spatial sampling of the window, we impose a maximum size of each subdomain at most equal to the smallest wavelength in each medium divided by a term of 15 , whereas the subwavelength apertures are sampled with a maximum spatial step of $5 \mathrm{~nm}$ to guarantee a sufficient number of elements to model the discontinuities.

Gold regions are then represented through a Drude model assisted by two Lorentz oscillators to fit the experimental data reported in [22]:

$$
\varepsilon_{m}(\omega)=\varepsilon_{\infty}-\frac{\omega_{D}^{2}}{\omega^{2}+j \omega \gamma_{D}}-\sum_{k=1}^{2} \frac{\delta_{k} \omega_{k}^{2}}{\omega^{2}-\omega_{k}^{2}+2 j \omega \gamma_{k}},
$$

where the high-frequency complex dielectric constant of the metal $\varepsilon_{\infty}$ is 5.398 , the plasma frequency $\omega_{D}$ and the collision frequency $\gamma_{D}$ of the Drude model are $1.398 \cdot 10^{16}$ and $1.033 \cdot 10^{14} \mathrm{rad} / \mathrm{s}$, respectively. The parameters describing the contribution of each Lorentz oscillator are the amplitude $\delta_{k}$, the resonant angular frequency $\omega_{k}$, and the damping constant $\gamma_{k}, k=1,2$, which are set according to [23]: $\delta_{1}=0.681, \omega_{1}=4.274 \cdot 10^{16} \mathrm{rad} / \mathrm{s}, \gamma_{1}=4.353 \cdot 10^{14} \mathrm{rad} / \mathrm{s}$, $\delta_{2}=1.861, \omega_{2}=5.225 \cdot 10^{16} \mathrm{rad} / \mathrm{s}$ and $\gamma_{2}=6.608 \cdot 10^{14} \mathrm{rad} / \mathrm{s}$.

The description of the numerical setup is completed by the definition of the excitation, which is a collection of CW plane waves in the wavelength range between 500 and $1000 \mathrm{~nm}$. This source field emerges from the top boundary of the computing cell with the wavevector $\mathbf{k}_{\text {in }}$ depicted in Figure 1, which is tilted of an angle $\theta$ with respect to the $z$-axis. Moreover, the polarization vector is assumed to be parallel to the grating wavevector in order to excite SPPs 
over the corrugated interfaces. The resulting fields are then collected on transmission and reflection line monitors placed on the bottom and on the top of the domain, $3 \mu \mathrm{m}$ far from the array, to compute the integral of the Poynting vector flowing through them.

2.1. Orthogonal Incidence. The exhaustive comprehension of the behavior of the free-standing system depicted in Figure 1 can be easily obtained by giving a first look into the transmission and reflection diagrams. Here the geometrical parameters are $w=200 \mathrm{~nm}, l=550 \mathrm{~nm}$, and $p=600 \mathrm{~nm}$. Moreover, the simulation has been performed by considering an input pulse illuminating orthogonally the whole system that is, the input wavevector is perpendicular to the grating plane. Transmission and reflection diagrams are reported in Figure 2(a).

It is important to underline that these spectra result by the application of periodic boundary conditions, that is, considering an infinite array of slits. Although this behavior is merely numerical, it is confirmed by experiments since it has been demonstrated that a number of sub-wavelength slits higher than 6 is enough to get the saturation of transmission to its threshold value [24]. The analysis of Figure 2(a) gives evidence to three distinctive regions of the diagram:

(i) a first region at higher wavelengths $(800 \mathrm{~nm})$, or lower frequencies where transmission shows a high maximum,

(ii) a deep of transmission localized around $650 \mathrm{~nm}$ corresponding to the center of a dip of transmission,

(iii) a sharp peak of transmission at lower wavelengths, or higher frequencies, around $600 \mathrm{~nm}$.

In other words, the presence of a periodicity seems to induce a dip for the light impinging orthogonally on the metal grating as occurs in photonic bandgap structures. Then, this minimum separates two well-defined states. The first state at lower energies $(\lambda=800 \mathrm{~nm})$ presents the character of a first-order Fabry-Pérot (FP) resonance acting in a cavity region bounded by two metal walls, defining the aperture, and two dielectric discontinuities between the homogeneous background and the metal-insulator-metal region [19]. The second resonant state of maximum of transmission at higher energies $(\lambda=611 \mathrm{~nm})$ is due to the presence of two SPP modes propagating over both the input and the output interfaces, giving rise to extraordinary optical transmission (EOT) [17]. In this case the input plane wave excites first a SPP mode over the input interface which couples with the symmetric SPP mode over the output interface through the array of slits. Finally SPPs start cooperating to the radiation of an outgoing plane wave with the same momentum, that is, wavelength, of the incident one. It is worth noting that the EOT state suffers the presence of absorption within gold, which already occurs around $600 \mathrm{~nm}$. For this reason this choice of parameters, that is, $w=200 \mathrm{~nm}, p=600 \mathrm{~nm}$, and $l=550 \mathrm{~nm}$, does not give evidence to a so-well defined maximum of transmission, which would be clear for an equivalent array of slits made on a silver film. Moreover, although this preliminary study is performed on a one-dimensional array, the same behavior can be found also in 2D arrays of gold rectangular patches [20].

More interesting for our purposes is the state of minimum of transmission at $\lambda=627.5 \mathrm{~nm}$. The inspection of Figure 2(b), which maps the absolute value of the total magnetic field at this wavelength, reveals that the electromagnetic energy is totally localized on the input interface, where a SPP mode is excited, so that the field is not transmitted to the output. The coupling mechanism between the incident light and the SPP resonating over the grating originates leaky modes, standing over the interface, which reradiate light to the reflection region with effects in the minimum of inhibited transmission through the aperture. It is of relevance to observe that energy is not flowing along the surface, as clearly shown by the inspection of the Poynting vector in Figure 2(b), thus demonstrating the stationary character of this SPP wave under the illumination of an infinite-extended plane wave.

From an analytical point of view, this minimum state occurs whenever the incident wavelength excites the SPP of the unperturbed air-metal interface, that is, for the first resonant order and under normal incidence:

$$
\lambda_{\mathrm{SPP}}=p,
$$

where $\lambda_{\text {SPP }}$ is the SPP wavelength given by the dispersion relation of SPPs propagating over a flat interface between the metal and the dielectric. The validity of this formulation is further proven by the initial hypothesis of sub-wavelength apertures, which does not alter significantly the dispersion relation of the SPP propagating on the flat metal layer.

At this stage it is important to point out that each dielectric-metal interface acts independently of the other one since the thickness of the metal layer is much higher than the skin depth of gold at the wavelength of interest. This statement can be proven by the examination of Figure 3(a), which details the spectral behavior of the same array of slits placed over a silica substrate with a refractive index $n_{\text {sub }}$ equal to 1.45 .

In this case it is clear that the matching condition in (2) is individually verified at the input and the output interfaces which are made of the same metal, but of different dielectrics, namely, air and silica. In particular, the minima occurs at $\lambda=626.5 \mathrm{~nm}$, almost equal to the one obtained in the previous symmetric case, and $\lambda=901.5 \mathrm{~nm}$, which corresponds to the excitation of a SPP mode on the goldsilica interface. This result is also confirmed by Figures 3(b) and $3(\mathrm{c})$ which plot the $x z$-section of the profile of the absolute value of the total magnetic field.

The only difference between the two minima of transmission resides on the presence of electromagnetic energy within the sub-wavelength aperture. With reference to Figure 3(c), the operation wavelength of the input field is no longer able to excite a resonant SPP mode over the air-gold interface. The field penetrates through the apertures and coherently excites a SPP mode over the gold-silica interface. Unlike the case in Figure 3(b), where the excitation of a resonant SPP over the first interface cuts off the waveguide mode within the 


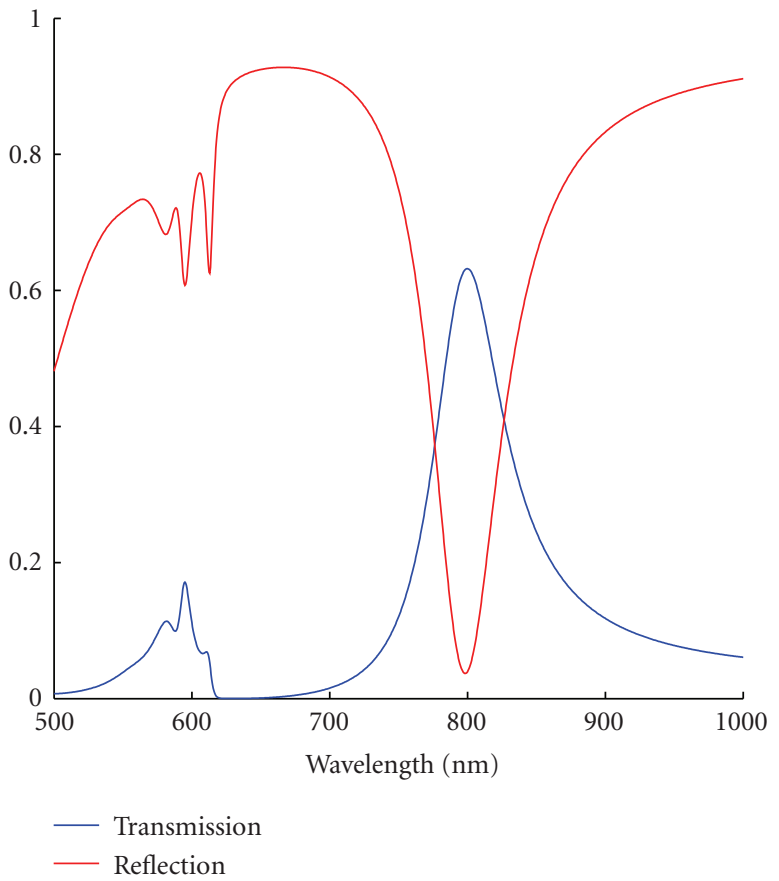

(a)

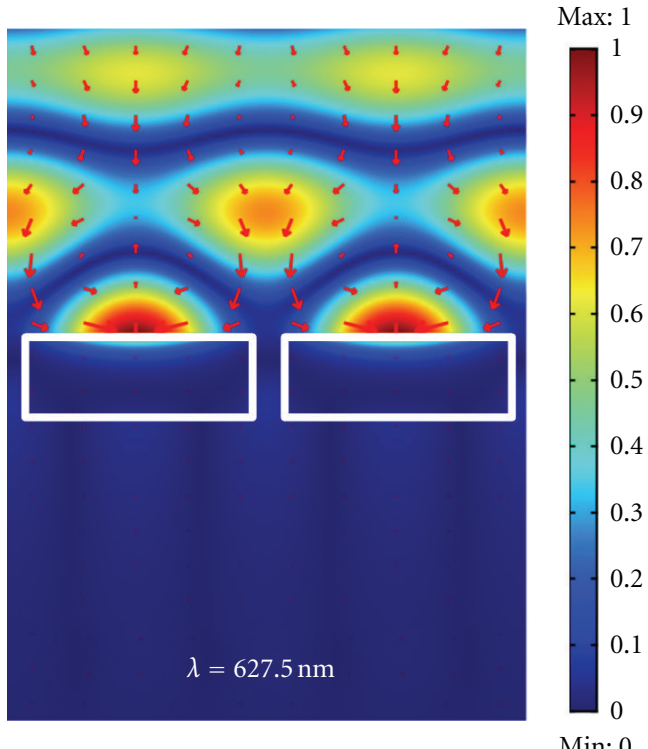

Min: 0

(b)

Figure 2: (a) Transmission and reflection diagrams of the free-standing array of metal stripes with $w=200 \mathrm{~nm}, p=600 \mathrm{~nm}, l=550 \mathrm{~nm}$. (b) Map of the transversal section of the absolute value of the magnetic field computed at $\lambda=627.5 \mathrm{~nm}$; red arrows display the direction of the Poynting vector.

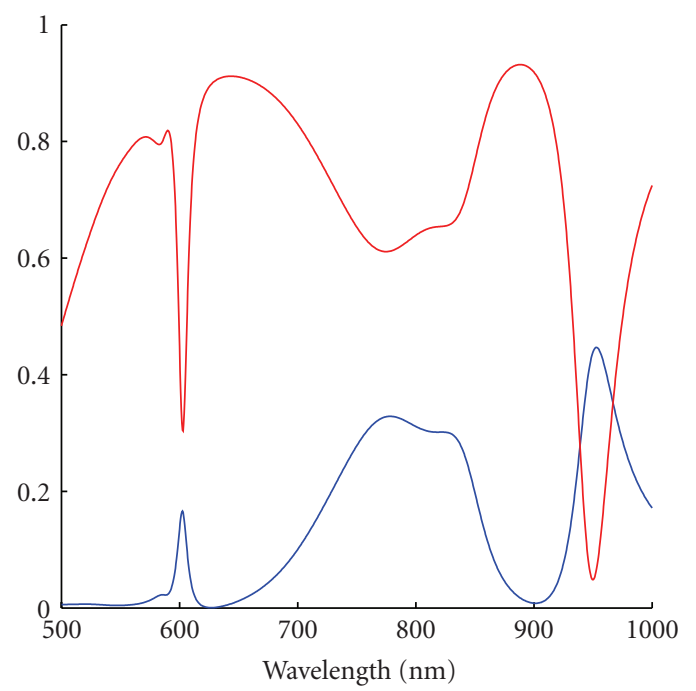

- Transmission
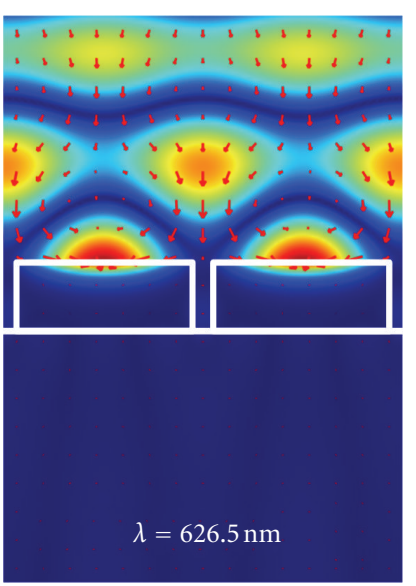

(b)

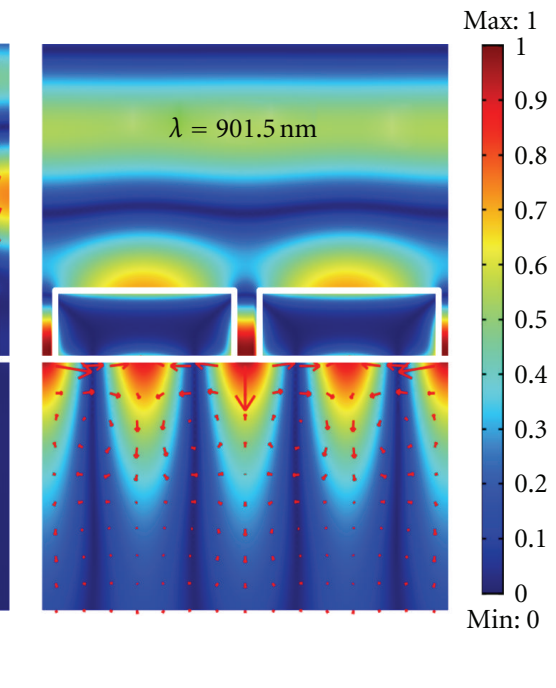

(c)

FIGURE 3: (a) Spectral response of an array of slits on the gold layer ( $w=200 \mathrm{~nm}, p=600 \mathrm{~nm}, l=550$ nm) placed over a silica substrate $\left(n_{\text {sub }}=1.45\right)$. Profile of the absolute value of the magnetic field computed at (b) $\lambda=626.5 \mathrm{~nm}$ and (c) $\lambda=901.5 \mathrm{~nm}$; red arrows express the direction of the Poynting vector. 
aperture, for $\lambda=901.5 \mathrm{~nm}$ the presence of electromagnetic energy in this region affects the transmission, which does not reach completely zero. This behavior is even more evident by looking at the direction of the Poynting vector in Figure 3(c), where the lengths of the arrows coming out from the apertures are higher than those related to the resonating SPPs over the output interface.

2.2. Nonorthogonal Incidence. The dependence of the angle of incidence $\theta$ on the position of the minimum of transmission is of crucial importance in the spectral behavior of the proposed structure. Equation (2) gives the condition for the excitation of SPP via grating coupling only in the case of orthogonal incidence and for a first order of resonance. If the light impinges with an angle $\theta$ (see Figure 1), the matching condition between wavevectors gives [25]

$$
k_{\mathrm{SPP}}=k_{0} \sin \theta \pm \Delta k_{x}
$$

where $\Delta k x=m G=m \cdot 2 \pi / p$ gives the contribution of any perturbation to the smooth metal-dielectric interface mediated by the apertures. Equation (3) can be rearranged to highlight the position of the minimum of transmission. In particular $\lambda_{\text {res, } m}$, that is, the free-space wavelength at which the external light excites a SPP mode on the metal grating, can be deduced using the following formulation:

$$
\lambda_{\text {res }, m}= \pm \frac{p}{m}\left(\sqrt{\frac{\varepsilon_{d} \varepsilon_{m}\left(\lambda_{\text {res }, m}\right)}{\varepsilon_{d}+\varepsilon_{m}\left(\lambda_{\text {res }, m}\right)}}-\sqrt{\varepsilon_{d}} \sin \theta\right) .
$$

The most important effect of the presence of a tilted light source resides in the splitting of the minimum of transmission in two different minima, which are very sensitive to the variation of this angle. An example of application of (4) to the case of an infinite array of slits on gold is reported in Figure 4. Here the minimum position is presented for the first two orders. Moreover the periodicity of the array of slits has been chosen equal to $700 \mathrm{~nm}$ in order to give clear evidence to the presence of a minimum of transmission for the wavelengths ranging between 500 and $1000 \mathrm{~nm}$. In addition, the structure is placed in a homogeneous medium, namely air, in order to keep the effect of the variation of the angle of incidence apart from the effect of a nonhomogeneous environment due to the presence of a dielectric substrate.

Since two different SPP modes decouple on the input interface of the metal grating, each mode has an effective refractive index, depending on the corresponding wavelength of excitation of the mode, satisfying (4). In particular

$$
n_{\mathrm{SPP}, m}^{ \pm}(\theta)=m \frac{\lambda_{\mathrm{res}, m}^{ \pm}(\theta)}{p} .
$$

Although this representation of the SPP mode is always valid, $n_{\text {SPP }}$ can range between well-defined limits. The lowest limit is due to the presence of ohmic losses within gold, since $\lambda_{\text {res, } m}^{-}$often occurs in the bulk-absorption region of the gold itself when $\theta$ increases. On the contrary, $n_{\mathrm{SPP}, m}^{+}$ can reach asymptotically the condition $n_{\mathrm{SPP}}(\theta=90 \circ)=$

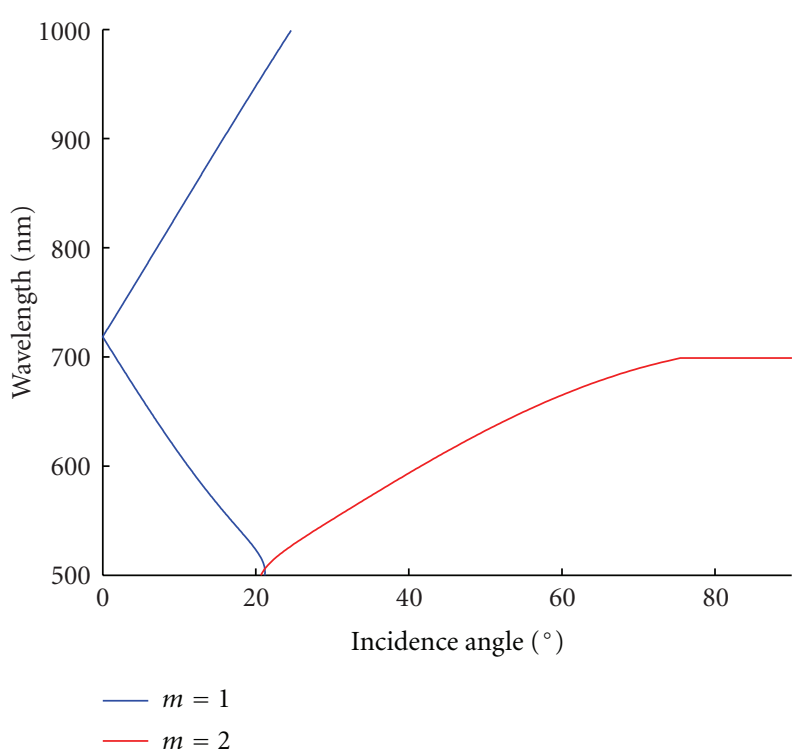

FIGURE 4: Spectral position of the first two orders of the gratingcoupling state as a function of the angle of incidence $\theta$ of the source field. The period of the array is now equal to $700 \mathrm{~nm}$.

$2 \cdot n_{\mathrm{SPP}}(\theta=0)$. This has results in the in-plane excitation of SPP modes which undergoes the well-known theory of the Bragg reflectors, forming the basis of the photonic crystal technology $[26,27]$.

Transmission and reflection diagrams computed on line monitors parallel to the metal-dielectric interfaces are reported in Figure 5. Here the gold film is $150 \mathrm{~nm}$ thick, the array of slits has a period of $700 \mathrm{~nm}$, and the width of the sub-wavelength apertures is $50 \mathrm{~nm}$. Moreover, we assume to probe the structure applying a plane wave with an infiniteextended wavefront, with angles of incidence equal to $0,5^{\circ}$, $10^{\circ}$, and $15^{\circ}$, respectively.

The excitation of two modes whose spectral positions depend on the incidence angle has effects in the formation of two different dips of transmission along the $z$-axis. The relative distance between these modes increases with the angle $\theta$, following the behavior displayed in Figure 4, down to the absorption region of gold, which is almost reached for $\theta=15^{\circ}$.

The $x z$-profile of the absolute value of the total magnetic field is shown in Figure 6. In this case we consider the same structure discussed above $(p=700 \mathrm{~nm}, w=150 \mathrm{~nm}$, $l=650 \mathrm{~nm}$ ) with an angle of incidence $\theta=10^{\circ}$ (for its definition in the reference system see the inset of Figure 6.

A first look to Figure 6 reveals that these plots are qualitatively comparable with that in Figure 2(b), valid in the case of orthogonal incidence over a free-standing array of slits with period equal to $600 \mathrm{~nm}$. In both cases the absolute value of the magnetic fields resembles a first order of resonance, since only one maximum of the electromagnetic field is always localized in the center of the metal elements.

Different considerations have to be carried out to explain the actual meaning of the direction of the Poynting vectors. Here the two SPP modes, due to the presence of a nonzero 


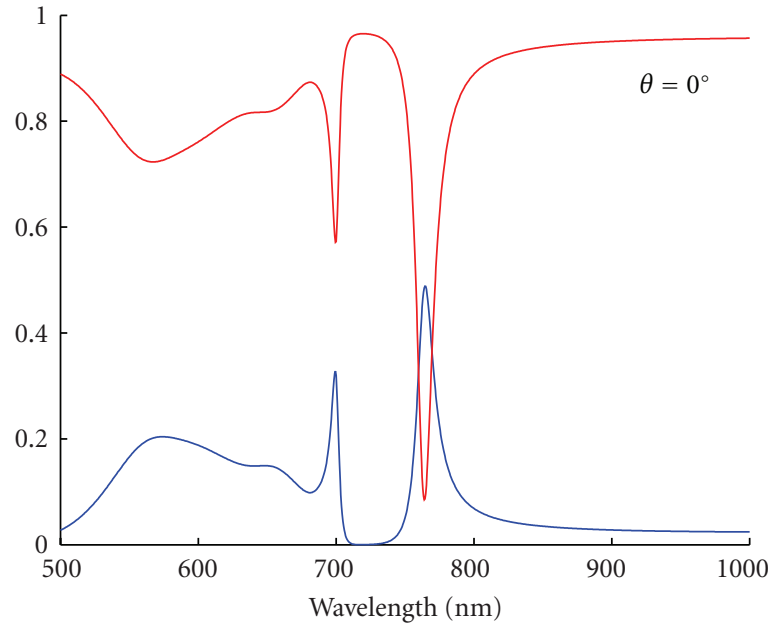

(a)

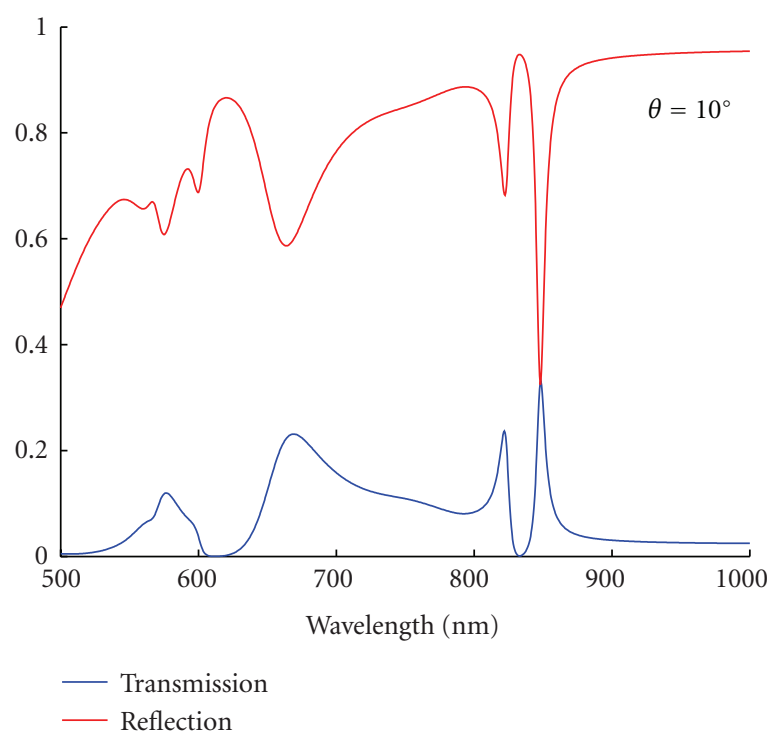

(c)

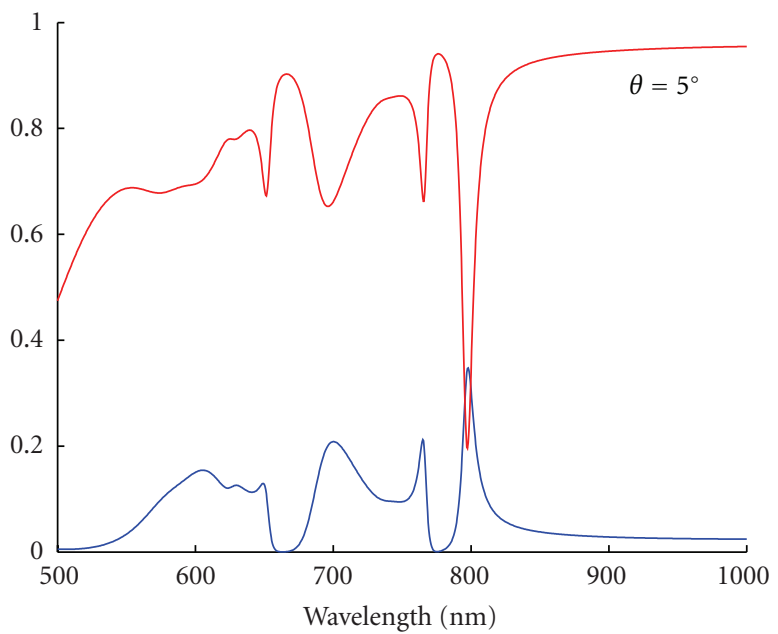

(b)

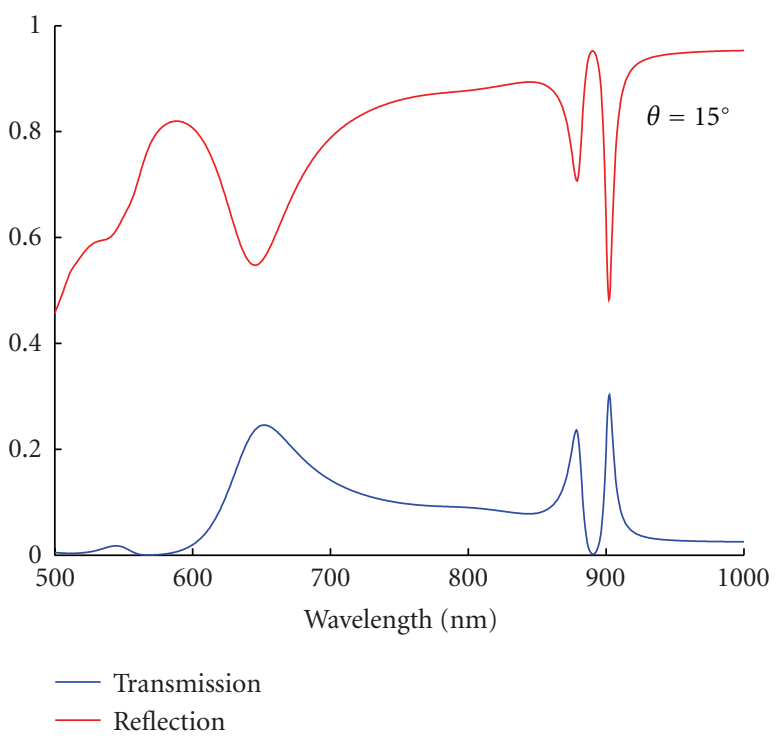

(d)

FIGURE 5: Parametric analysis of the transmission and the reflection diagrams of a free-standing array of gold strips computed for (a) $\theta=0^{\circ}$, (b) $\theta=5^{\circ}$, (c) $\theta=10^{\circ}$, and (d) $\theta=15^{\circ}$. The geometrical parameters are $p=700 \mathrm{~nm}, w=150 \mathrm{~nm}$, and $l=650 \mathrm{~nm}$.

angle of incidence, are counterpropagating over the metaldielectric interface. The up mode at $\lambda=833.61 \mathrm{~nm}$ has an opposite direction with reference to the $x$-component of the exciting wavevector $\mathbf{k}_{\text {in }}$. Taking this mode to the up edge of the range of existence of $n_{\mathrm{SPP}, 1}^{+}$, that is, for inplane excitation $\left(\theta=90^{\circ}\right)$, we will find that the SPP is completely reflected accordingly to Bragg's law. As known in SPP bandgap structures [28], the operation wavelength in the medium has to be substituted by the SPP wavelength coming out by the dispersion equation of the SPP propagating over the smooth metal-dielectric interface. Quantitatively, this condition occurs at $\lambda_{\mathrm{res}, 1}^{+}\left(\theta=90^{\circ}\right)=1403.5 \mathrm{~nm}$. At the same time, the down mode propagates with the same direction of the $x$-component of $\mathbf{k}_{\text {in }}$, opposite to that of the up mode. As observed previously, the lowest edge for the propagation of this mode, that is, at $\theta=90^{\circ}$, falls down to the absorption region of the metal and thus cannot be estimated with accuracy.

\section{Equivalent Structure}

Once the physics below the excitation of SPPs over a metal gratin has been explained, we go on with the analysis of realistic systems, invoking an equivalence of structures to better display that the punctual minima of transmission broaden to actually form an effective stop band for light.

With reference to Figure 7, the original structure, illuminated by a nonorthogonal light beam, is studied through an equivalent structure with effective period and substrate, 


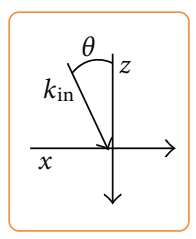

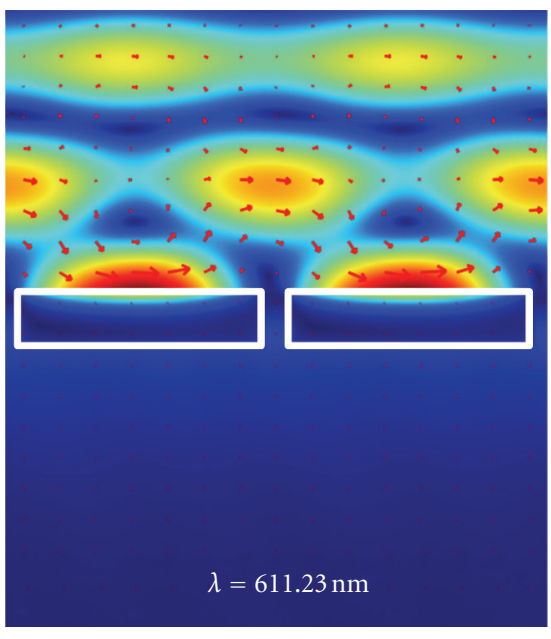

(a)

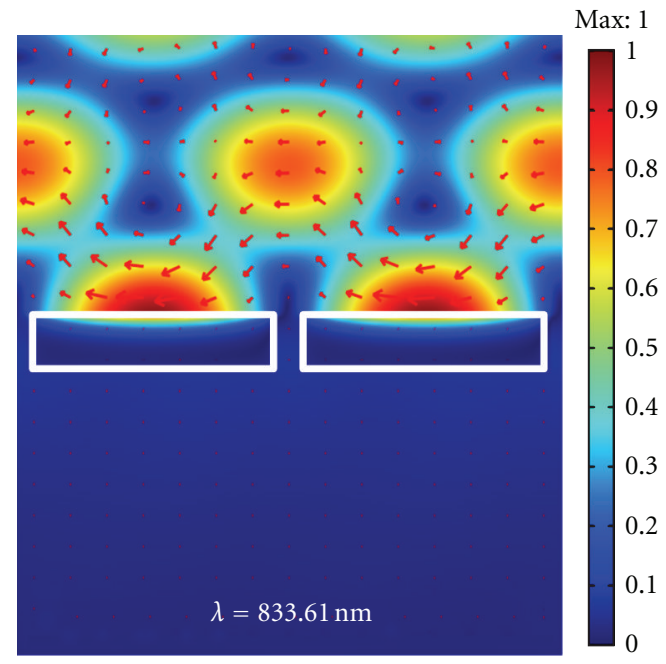

Min: 0

(b)

Figure 6: Absolute value of the magnetic field for the structure described in Figure 5 with $\theta=10^{\circ}$ computed at (a) $\lambda=611.23$ nm and (b) $\lambda=833.61 \mathrm{~nm}$. Red arrows in both plots express the Poynting vector direction.

but orthogonally excited. The choice of the equivalent parameters depends on the couple of values $(m, \theta)$ of the original system: the effective period $p_{\mathrm{eq}}$ is chosen to reproduce the SPP excitation of the $m$ th down mode at $\lambda_{\text {res, } m}^{-}(\theta)$, whereas the effective substrate is found to model the effect of the $m$ th up mode at $\lambda_{\text {res }, m}^{+}(\theta)$. In this way the two modes due to the non-zero angle of incidence behave as two SPP modes resonating over the two interfaces of the asymmetric structure: the down mode at $\lambda_{\text {res, } m}^{-}(\theta)$ over the metal-cover interface and the up mode at $\lambda_{\text {res }, m}^{+}(\theta)$ over the metal-substrate interface. In this hypothesis, it is clear that the validity of the equivalence showed in Figure 7 is guaranteed at the wavelengths where the up and down modes are excited for a particular value of the angle of incidence $\theta$, that is, at $\lambda_{\text {res, } m}^{-}(\theta)$ and $\lambda_{\text {res, } m}^{-}(\theta)$. For convenience we suppose to cover the equivalent structure with air.

Since the expression of the equivalent period is merely dependent on $\lambda_{\text {res, } m}^{-}(\theta)$, we can make (2) more explicit and merge this result to (4), thus obtaining

$$
\frac{p_{\mathrm{eq}}}{m}=\frac{\lambda_{\min , m}^{-}(\theta)}{\sqrt{\varepsilon_{m}\left(\lambda_{\min , m}^{-}(\theta)\right) /\left(1+\varepsilon_{m}\left(\lambda_{\min , m}^{-}(\theta)\right)\right)}} .
$$

In a similar way, but referring to the metal-substrate interface of a $p_{\text {eq }}$-periodic grating, we can find the analytical expression of the dielectric function of the equivalent substrate:

$$
\varepsilon_{d, \text { eq }}=\frac{\varepsilon_{m}\left(\lambda_{\min , m}^{+}(\theta)\right)\left(m\left(\lambda_{\min , m}^{+}(\theta) / p_{\mathrm{eq}}\right)\right)^{2}}{\varepsilon_{m}\left(\lambda_{\min , m}^{+}(\theta)\right)-\left(m\left(\lambda_{\min , m}^{+}(\theta) / p_{\mathrm{eq}}\right)\right)^{2}} .
$$

It is important to underline that this expression can be applied for each wavelength of the range of interest,

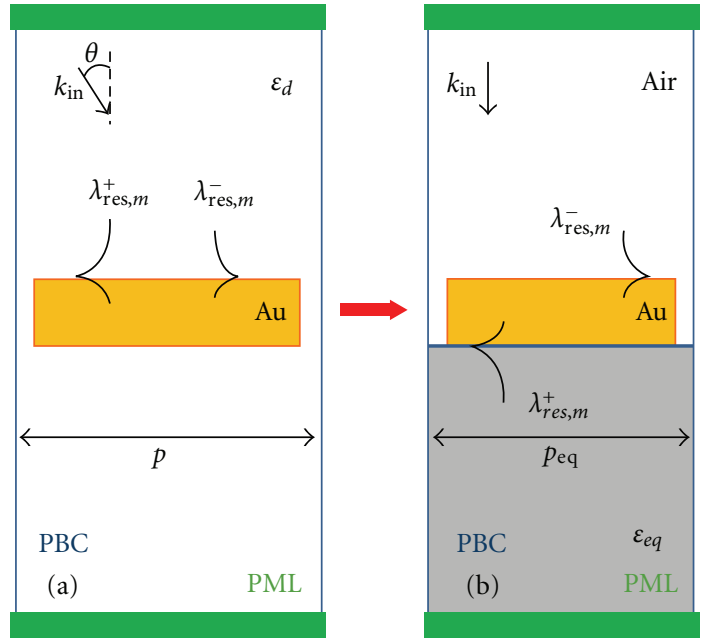

Figure 7: Sketch of (a) the original array of slits and (b) the corresponding equivalent structure with orthogonal excitation. SPP modes are also reported on the proper metal-dielectric interfaces.

since it does not interact with the equivalent down mode resonating over the metal-cover interface (see Paragraph 2.1). Nevertheless, it only has a physical meaning for values

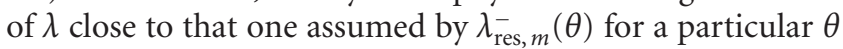
value. Moreover, the formulations (6) and (7) do not include the metal thickness, since it does not alter considerably the SPP resonance over the grating when the thickness itself is much larger than the skin depth of metal, that is, as in the presented cases.

The analytical formulation of the parameters involved in the model of the equivalent system allows the detailed extraction of the actual values to be implemented. Table 1 
TABLE 1: Resonant wavelengths corresponding to the excitation of the SPP modes over the air-metal interface of the array of gold stripes with $p=700 \mathrm{~nm}$ and corresponding parameters for the equivalent structures.

\begin{tabular}{lcccc}
\hline$\theta$ & $\lambda_{\text {res }, 1}^{+}(\mathrm{nm})$ & $\lambda_{\text {res }, 1}^{-}(\mathrm{nm})$ & $p_{\mathrm{eq}}(\mathrm{nm})$ & $\varepsilon_{\mathrm{eq}}(\theta)$ \\
\hline $0^{\circ}$ & 718.49 & 718.49 & 700 & 1 \\
$5^{\circ}$ & 775.72 & 663.08 & 641.02 & 1.3814 \\
$10^{\circ}$ & 833.61 & 611.23 & 583.88 & 1.9071 \\
$15^{\circ}$ & 891.29 & 565.12 & 530.07 & 2.6169 \\
\hline
\end{tabular}

reports the resonant wavelengths of the SPP modes of the first order for the cases already presented in Figure 5 and the equivalent parameters obtained by the application of (6) and (7).

The application of these parameters to the equivalent structure allows the comparison of spectra with those already exposed in Figure 5. In particular Figure 8 reports this result in terms of transmission profile for the case in which the original structure is illuminated by a light beam with an angle of incidence equal to $5^{\circ}, 10^{\circ}$, and $15^{\circ}$.

Before going through the inspection of Figure 8 , it is of relevance to notice that the equivalence described by (6) and (7) is suitable only in a wavelength range close to the SPP resonances, so that the comparison of diagrams is expected to show significant differences far from the resonant wavelengths of the two SPP modes. As a result, the diagrams in Figures 8(a) and 8(b) show a good agreement between SPP resonances found by the equivalent structure on those of the original one. Table 2 reports the comparison of the resonant wavelengths resulting from the analysis of the original structure and the equivalent ones with angles of incidence of the source field equal to $5^{\circ}, 10^{\circ}$ and $15^{\circ}$.

In particular, it is evident a good matching between the resonant wavelengths of the systems corresponding to the cases of $\theta=5^{\circ}$ and $\theta=10^{\circ}$, with a relative error smaller than $0.7 \%$. On the other hand, Figure 8 (c) gives evidence to an overestimation of the resonant wavelengths with respect to the one obtained with the original structure. The nature of this phenomenon can be found in the presence of losses induced by the metal on the down mode which raises close to the absorption band of gold. This mode only affects the expression of the equivalent period, according to (6), thus inducing an error in its model. Then, the measurement uncertainty due to high absorption interferes with the definition of the higher resonance, which depends itself on the down mode. These considerations allow us to confirm that the range of validity of $\theta$ for the proposed equivalence of structures is determined by the relative position of the down mode with respect to the absorption band of the metal, in a similar way to what noticed in the previous paragraph with reference to (5). This implies that, if perfect metals are considered, that is, any ohmic-loss mechanism is taken into account, the equivalence is always verified. Further simulations prove the equivalence of results under this hypothesis with negligible relative errors (lower than $0.06 \%$ ) for each angle of incidence.
Moreover, although this is not of interest for this paper, which is mostly focused on the excitation of SPP modes, it is important to show that the peaks of resonance of high transmission at the lowest edges of the up modes disappear in all the proposed cases. This occurrence has a physical meaning ascribable to the character of this kind of resonance. In other words, since this peak of high transmission is due to the presence of coupled SPPs propagating on both input and output interfaces with the same phase laws, it can be only found in symmetric systems [17]. The presence of an equivalent substrate cuts off this resonant mode with results in the transmission profiles.

Figure 9 maps the absolute value of the resonant magnetic field obtained by the analysis of the equivalent structure related to the case of $\theta=10^{\circ}$.

The analysis of Figure 9 highlights the different behaviors of the two resonating modes. As expected, the down mode at $\lambda_{\text {res, } 1}^{-}=611.23 \mathrm{~nm}$ resonates as a SPP mode over the airgold interface, inducing an efficient stop condition for the energy impinging on the grating, in a manner similar to that in Figure 6(a), valid for the original system with $\theta=10^{\circ}$. For this reason all the down modes have a depth comparable to the ones obtained by the response of the original structures, within a numerical tolerance. Conversely, the up mode changes its nature becoming a SPP mode resonating over the gold-substrate interface. As already observed in Paragraph 2.1, the excitation of a SPP mode over the output interface does not switch off the resonance within the sub-wavelength aperture, thus allowing light to reach the transmission. This aspect does not allow the achievement of the zerotransmission condition for the up mode, as comes out from Figure 8.

Another important aspect regards the direction of the Poynting vector related to the resonant modes over the two interfaces. As shown previously, the presence of a non-zero angle of incidence induces the excitation of two counterpropagating modes at different wavelengths in the original system. By looking at the red arrows in Figure 9, the energy of the SPP modes stands over the grating without a dominant flow direction. This is the result of the orthogonal excitation of the slit array which produces a stationary mode reradiating to the reflection. Nevertheless, it is important to underline that this is only due to the kind of excitation used in our numerical measurements, that is, an infinite-extended plane wave. When the source is spatially bounded, assuming, for instance, a Gaussian shape, each mode at $\lambda_{\text {res, } m}^{-}$and $\lambda_{\text {res, } m}^{-}$ stationary resonates with the profile in Figure 9 only in the bound region effectively covered by the incidence of the input beam. Figure 10 shows the magnetic field response of the structure in Figure 9 under the application of a Gaussian-shaped input pulse able to excite the down mode at $\lambda=611.23 \mathrm{~nm}$.

The analysis of the Poynting vector directions in Figure 10 proves that, since each of these modes is the superposition of the two counterpropagating modes, these components decouple propagating in opposite direction through the side regions. Moreover, the propagation length of such modes has been found the same of the original structure, since the main loss mechanism suffered by SPPs 


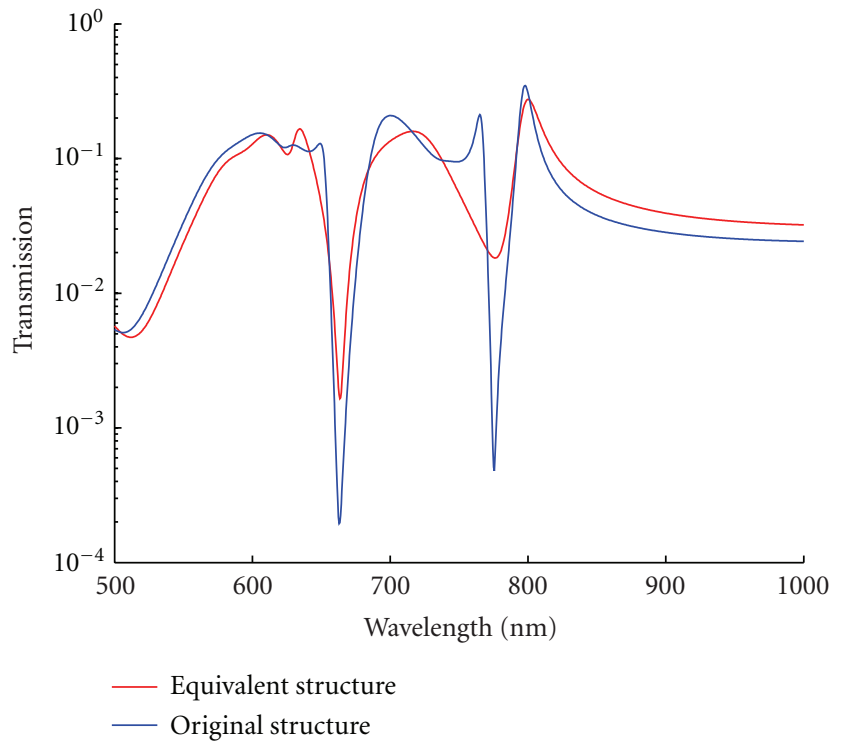

(a)

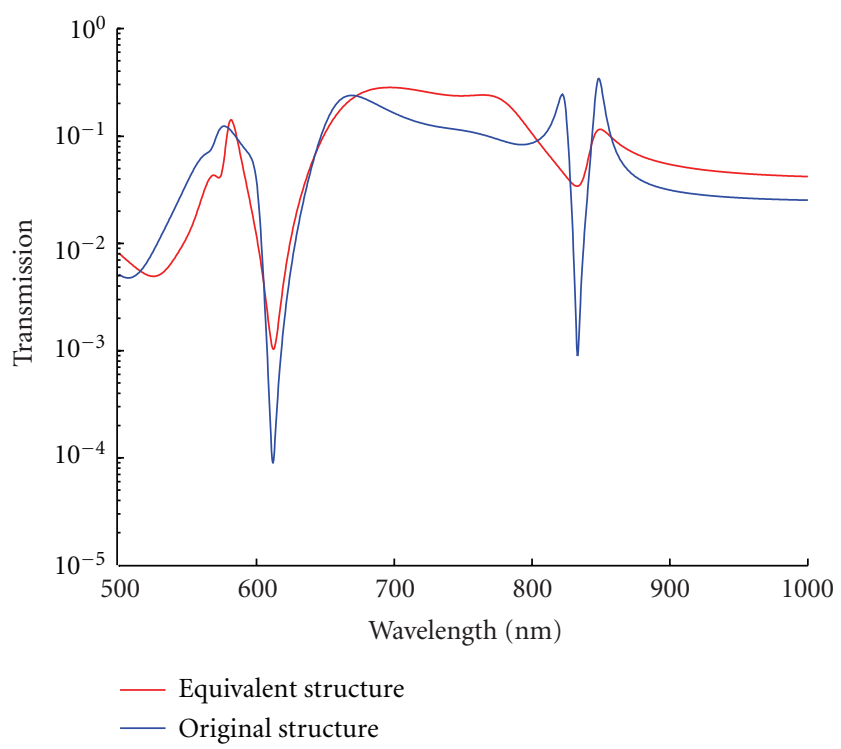

(b)

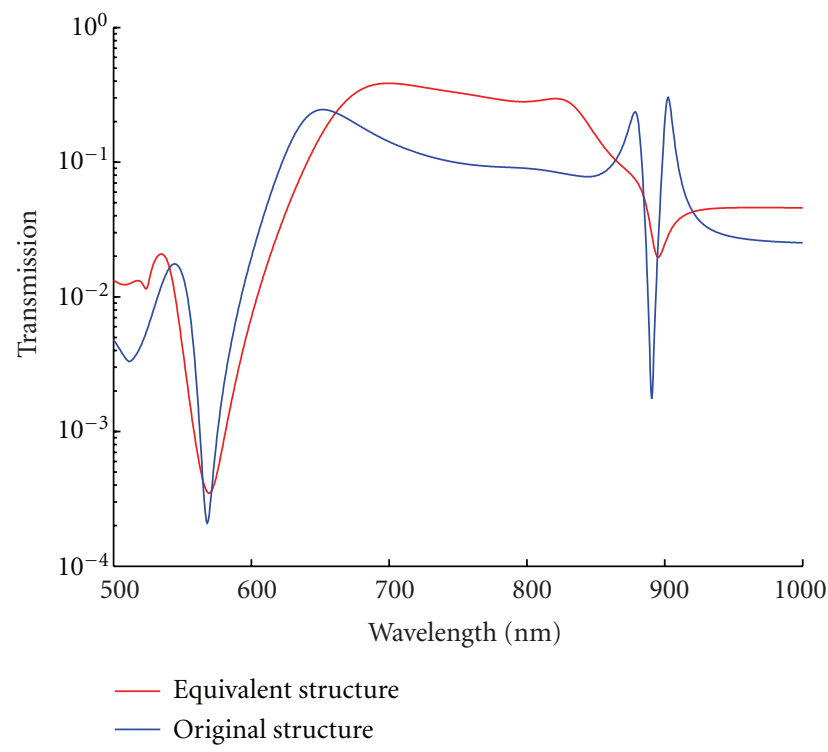

(c)

FiguRE 8: Blue lines report the transmission diagrams already presented in Figure 5 in logarithmic scale, whereas red lines display on the same scale the results obtained by the equivalent structures with the parameters listed in Table 1 . The angles of incidence of the input light for the original arrays are (a) $\theta=5^{\circ}$, (b) $\theta=10^{\circ}$, and (c) $\theta=15^{\circ}$.

TABLE 2: Comparison of the resonant wavelengths obtained by the analysis of the original structure with different angles of incidence and its equivalent counterparts.

\begin{tabular}{|c|c|c|c|c|}
\hline \multirow[b]{2}{*}{$\theta$} & \multicolumn{2}{|c|}{ Original structure } & \multicolumn{2}{|c|}{ Equivalent structure } \\
\hline & $\lambda_{\mathrm{res}, 1}^{+}(\mathrm{nm})$ & $\lambda_{\text {res }, 1}^{-}(\mathrm{nm})$ & $\lambda_{\mathrm{res}, 1}^{+}(\mathrm{nm})$ & $\lambda_{\text {res }, 1}^{-}(\mathrm{nm})$ \\
\hline $5^{\circ}$ & 775.72 & 663.08 & 776.15 & 663.51 \\
\hline $10^{\circ}$ & 833.61 & 611.23 & 832.78 & 612.36 \\
\hline $15^{\circ}$ & 891.29 & 565.12 & 895.42 & 569.08 \\
\hline
\end{tabular}




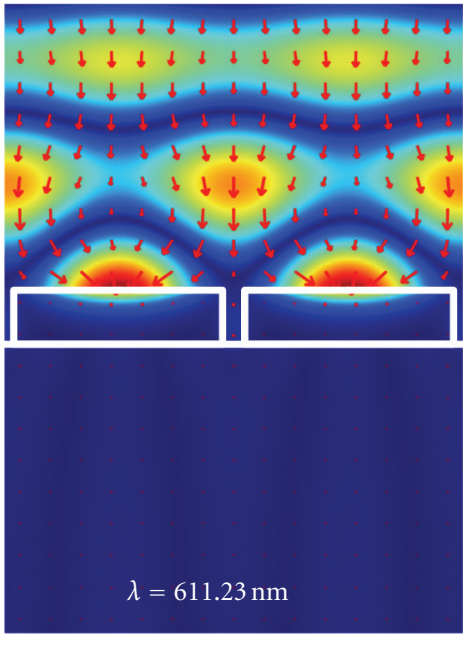

(a)

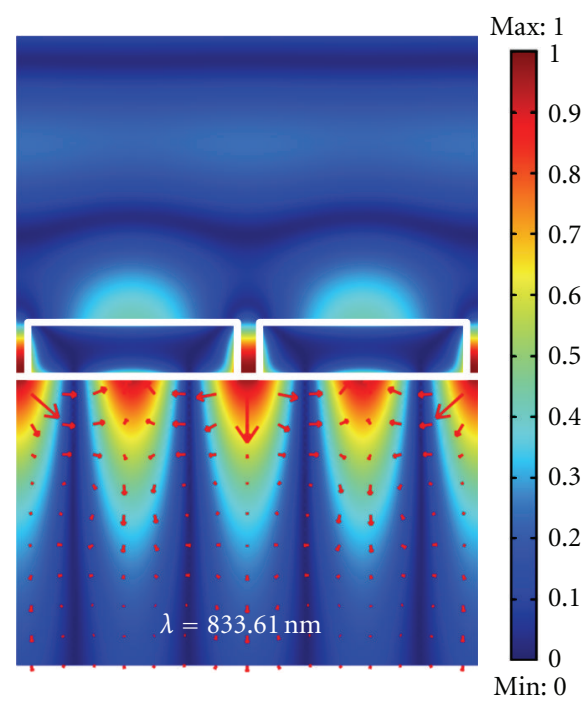

(b)

Figure 9: Absolute value of the magnetic field for the equivalent system applied to the case of $\theta=10^{\circ}\left(p_{\mathrm{eq}}=583.88 \mathrm{~nm}\right.$ and $\left.\varepsilon_{\mathrm{eq}}=1.9071\right)$. Computations are performed at (a) $\lambda=611.23 \mathrm{~nm}$ and (b) $\lambda=833.61 \mathrm{~nm}$. Red arrows indicate the Poynting vector direction.

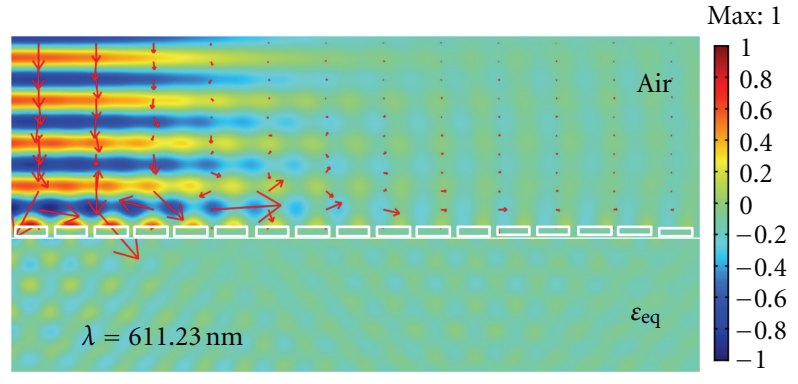

Figure 10: Magnetic field component $H_{z}$ at $\lambda=611.23 \mathrm{~nm}$ of the equivalent system with the parameters in Figure 9 applied to the case of $\theta=10^{\circ}$. Exploiting the even symmetry of the structure, the left side of the magnetic field is the mirrored replica of this plot. Red arrows indicate the direction of the Poynting vector. In this case the source has a Gaussian-shaped spatial profile.

is due to radiation phenomena owing to the presence of the sub-wavelength apertures.

The equivalence of structures allows an easy comprehension of the actual bandgap formation in slit arrays subjected to an out-of-plane excitation, although the effective incidence of a source field over a metal grating would only generate a dip of transmission following the momentum conservation in (3).

With reference to the experimental demonstration in [4], a measurement setup able to excite SPP modes over periodic arrangements of metal elements for sensing purposes makes use of microscope objectives to focus a white light over the grating. As a consequence, the impinging light is in a cone more than $10^{\circ}$ wide. This produces a measured effect of an enlargement of the complete-reflection band.
Since the proposed equivalence of structures is still valid for two-dimensional arrays of metal square elements, as the two polarizations act independently, experiencing the same periodicity, the simple original structure can be seen as a weighted superposition of results coming out by the analysis of several equivalent systems. Exploiting the mathematical formalism given by (6) and (7), we find that the equivalent structures for the air-metal interface have periods ranging in an interval of about $100 \mathrm{~nm}$, with a maximum shift of the dielectric constant equal to 0.87 . It means that the original system with practical normal excitation can be seen as made of many different structures with a perfectly orthogonal excitation. In this way the broadening of the stop band for input light becomes clear, although the physical meaning resides in the excitation of several SPP up and down modes over the grating. Thus, we can consider the effective opening of a finite bandgap for light impinging orthogonally on actual systems.

Moreover, this equivalence is also helpful to understand the capability of these arrangements of metal elements in solar cells designed to improve the energy absorption. In fact, a single system excited by sunlight with random incident angles behaves as a set of structures, each one with its equivalent period and substrate, with effects in the broadening of the bandgap of transmitted energy, very suitable for back reflectors.

\section{Conclusions}

In this paper we have demonstrated through numerical experiments that a non-zero angle of incidence induces the formation of an effective finite bandgap in practical systems. From a physical point of view, out-of-plane incidences over metal gratings made of periodic arrangements of slits on metal layers generate two SPP modes whenever the 
momentum conservation is fulfilled. Such modes propagate over the metal grating in opposite directions and degenerate in a standing wave if light impinges orthogonally with an infinite wavefront. By exploiting the independence of surface modes over the two interfaces of the metal film, we define an equivalence of structures able to convert the counterpropagating SPP modes, due to the non-zero incident angle of the source field, in two spatially separated SPP modes, generated by an orthogonal source field and propagating at the same wavelength of those of the original system, but on different interfaces. The equivalence of structures has been applied to the case of a realistic measurement setup, where the input light is in a cone at least $10^{\circ}$ wide. In this case the behaviour of the actual structure can be described by a set of equivalent systems ideally illuminated by a perfectly orthogonal excitation with effective periods ranging in an interval of about $100 \mathrm{~nm}$, with a maximum shift of the dielectric constant of the equivalent substrate equal to 0.87 . In this way we prove that realistic systems show effective bandgaps owing to the excitation of several SPP modes.

\section{Acknowledgments}

The authors acknowledge the CINECA Award for the proposed project SPMAPS — Surface Plasmon Management in Active and Passive Systems - 2011 and for the availability of high-performance computing resources and support.

\section{References}

[1] R. Ameling, L. Langguth, M. Hentschel, M. Mesch, P. V. Braun, and H. Giessen, "Cavity-enhanced localized plasmon resonance sensing," Applied Physics Letters, vol. 97, no. 25, Article ID 253116, 2010.

[2] Y. T. Chang, Y. C. Lai, C. T. Li, C. K. Chen, and T. J. Yen, "A multi-functional plasmonic biosensor," Optics Express, vol. 18, no. 9, pp. 9561-9569, 2010.

[3] M. Grande, R. Marani, F. Portincasa et al., "Asymmetric plasmonic grating for optical sensing of thin layers of organic materials," Sensors and Actuators, B, vol. 160, no. 1, pp. 10561062, 2011.

[4] M. Grande, M. A. Vincenti, T. Stomeo et al., "Experimental demonstration of a novel bio-sensing platform via plasmonic band gap formation in gold nano-patch arrays," Optics Express, vol. 19, no. 22, pp. 21385-21395, 2011.

[5] M. Grande, M. A. Vincenti, T. Stomeo et al., "Twodimensional plasmonic nano-structures for linear and nonlinear regimes," in Proceedings of the Micro- and nano-photonic materials and devices (MINAP '12), pp. 73-76, Trento, Italy, January 2012.

[6] J. Bravo-Abad, F. J. García-Vidal, and L. Martín-Moreno, "Wavelength de-multiplexing properties of a single aperture flanked by periodic arrays of indentations," Photonics and Nanostructures - Fundamentals and Applications, vol. 1, no. 1, pp. 55-62, 2003.

[7] R. Marani, V. Marrocco, M. Grande, G. Morea, A. D’Orazio, and V. Petruzzelli, "Enhancement of extraordinary optical transmission in a double heterostructure plasmonic bandgap cavity," Plasmonics, vol. 6, no. 3, pp. 469-476, 2011.
[8] D. J. Bergman and M. I. Stockman, "Surface plasmon amplification by stimulated emission of radiation: quantum generation of coherent surface plasmons in nanosystems," Physical Review Letters, vol. 90, no. 2, Article ID 027402, 4 pages, 2003.

[9] M. A. Noginov, G. Zhu, A. M. Belgrave et al., "Demonstration of a spaser-based nanolaser," Nature, vol. 460, no. 7259, pp. 1110-1112, 2009.

[10] R. Marani, A. D’Orazio, V. Petruzzelli et al., "Gain-assisted extraordinary optical transmission through periodic arrays of subwavelength apertures," New Journal of Physics, vol. 14, Article ID 013020, 2012.

[11] K. R. Catchpole and A. Polman, "Plasmonic solar cells," Optics Express, vol. 16, no. 26, pp. 21793-21800, 2008.

[12] M. Losurdo, M. M. Giangregorio, G. V. Bianco, A. Sacchetti, P. Capezzuto, and G. Bruno, "Enhanced absorption in $\mathrm{Au}$ nanoparticles/a-Si:H/c-Si heterojunction solar cells exploiting Au surface plasmon resonance," Solar Energy Materials and Solar Cells, vol. 93, no. 10, pp. 1749-1754, 2009.

[13] V. Marrocco, R. Marani, M. Grande et al., "Modification of the scattering of silver nanoparticles induced by Fabry-Pérot resonances rising from a finite Si layer," Journal of Optics, vol. 13, no. 1, Article ID 015004, 2011.

[14] D. Dregely, R. Taubert, J. Dorfmüller, R. Vogelgesang, K. Kern, and H. Giessen, "3D optical Yagi-Uda nanoantenna array," Nature Communications, vol. 2, no. 1, article 267, 2011.

[15] T. Dattoma, M. Grande, R. Marani, G. Morea, V. Marrocco, and A. D'Orazio, "Resonancewavelength dependence and mode formation in gold nanorod optical antennas with finite thickness," Progress In Electromagnetics Research B, no. 30, pp. 337-353, 2011.

[16] T. W. Ebbesen, H. J. Lezec, H. F. Ghaemi, T. Thio, and P. A. Wolff, "Extraordinary optical transmission through subwavelenght hole arrays," Nature, vol. 391, no. 6668, pp. 667$669,1998$.

[17] J. A. Porto, F. J. García-Vidal, and J. B. Pendry, "Transmission resonances on metallic gratings with very narrow slits," Physical Review Letters, vol. 83, no. 14, pp. 2845-2848, 1999.

[18] D. F. P. Pile, T. Ogawa, D. K. Gramotnev et al., "Twodimensionally localized modes of a nanoscale gap plasmon waveguide," Applied Physics Letters, vol. 87, no. 26, Article ID 261114, pp. 1-3, 2005.

[19] D. De Ceglia, M. A. Vincenti, M. Scalora, N. Akozbek, and M. J. Bloemer, "Plasmonic band edge effects on the transmission properties of metal gratings," AIP Advances, vol. 1, no. 3, Article ID 032151, 2011.

[20] R. Marani, M. Grande, V. Marrocco et al., "Plasmonic bandgap formation in two-dimensional periodic arrangements of gold patches with subwavelength gaps," Optics Letters, vol. 36, no. 6, pp. 903-905, 2011.

[21] COMSOL Multiphysics, http://www.comsol.com.

[22] E. D. Palik, Handbook of Optical Constants of Solids, Academic Press, San Diego, Calif, USA, 1998.

[23] J. M. McMahon, J. Henzie, T. W. Odom, G. C. Schatz, and S. K. Gray, "Tailoring the sensing capabilities of nanohole arrays in gold films with Rayleigh anomaly-surface plasmon polaritons," Optics Express, vol. 15, no. 26, pp. 18119-18129, 2007.

[24] Y. S. Jung, J. Wuenschell, H. K. Kim, P. Kaur, and D. H. Waldeck, "Blue-shift of surface plasmon resonance in a metal nanoslit array structure," Optics Express, vol. 17, no. 18, pp. 16081-16091, 2009.

[25] H. Raether, Surface Plasmons on Smooth and Rough Surfaces and on Gratings, Springer, Berlin, Germnay, 1986. 
[26] K. Sakoda, Optical Properties of Photonic Crystals, Springer, Berlin, Germany, 2005.

[27] J. D. Joannopoulos, S. G. Johnson, J. N. Winn, and R. D. Meade, Photonic Crystals-Molding the Flow of, Princeton University Press, Princeton, NJ, USA, 2008.

[28] S. I. Bozhevolnyi, J. Erland, K. Leosson, P. M. W. Skovgaard, and J. M. Hvan, "Waveguiding in surface plasmon polariton band gap structures," Physical Review Letters, vol. 86, no. 14, pp. 3008-3011, 2001. 

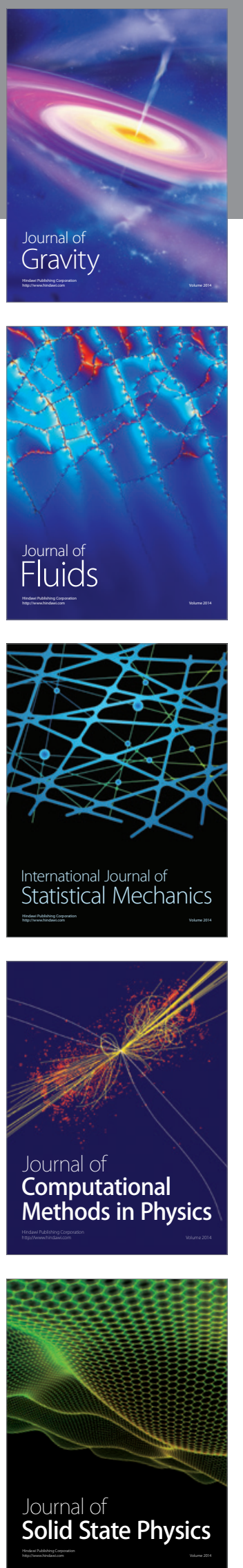

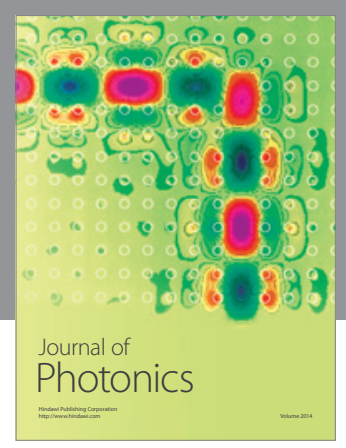

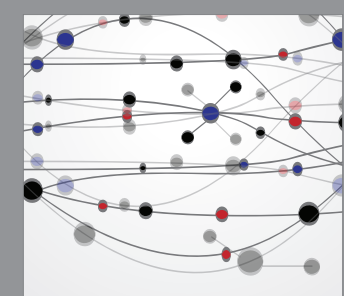

The Scientific World Journal
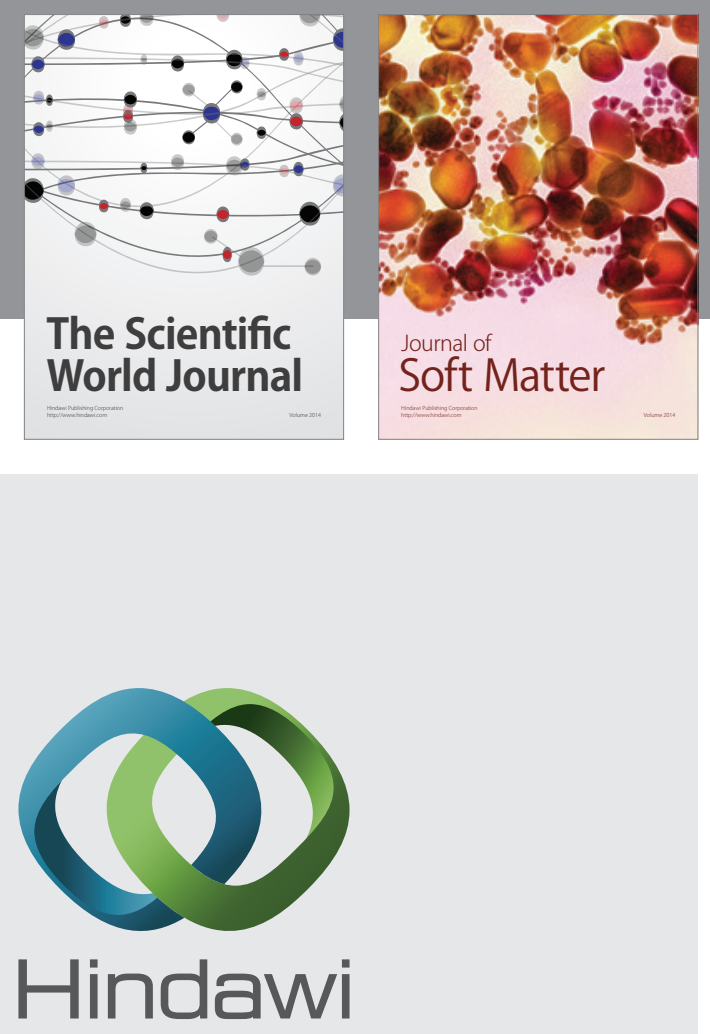

Submit your manuscripts at

http://www.hindawi.com
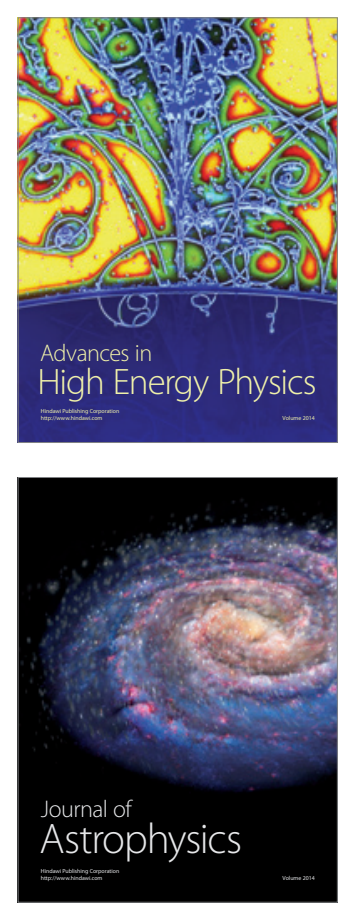
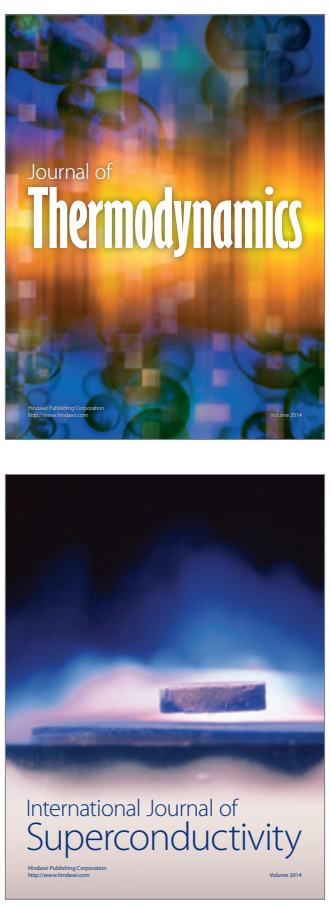
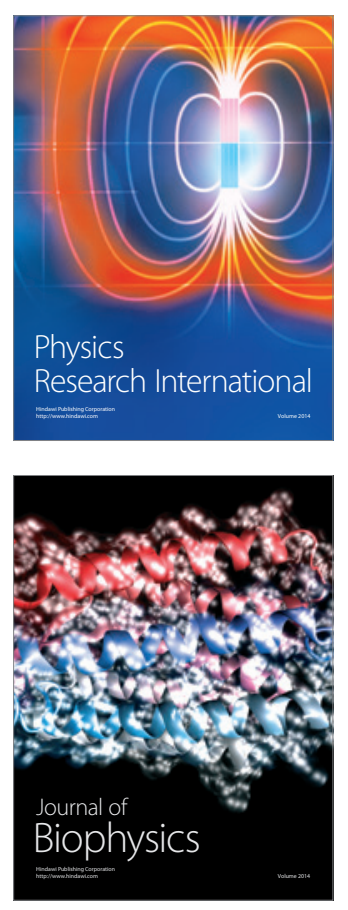
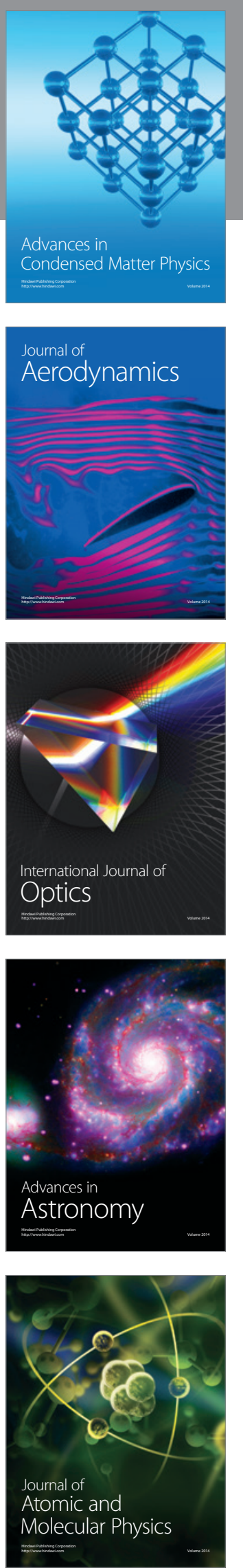